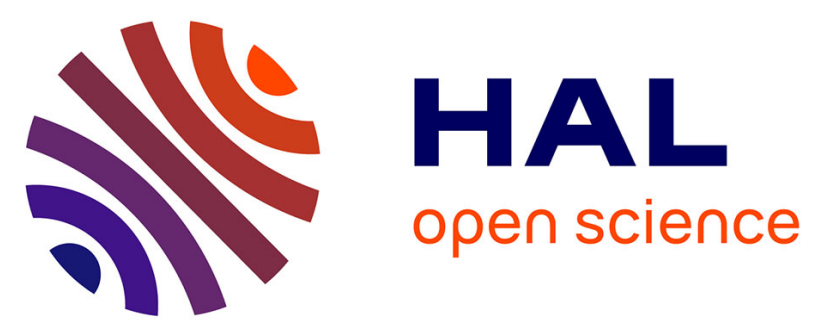

\title{
On the FPGA-based implementation of a flexible waveform from a high-level description: Application to LTE FFT case study
}

Mai-Thanh Tran, Matthieu Gautier, Emmanuel Casseau

\section{- To cite this version:}

Mai-Thanh Tran, Matthieu Gautier, Emmanuel Casseau. On the FPGA-based implementation of a flexible waveform from a high-level description: Application to LTE FFT case study. EAI International Conference on Cognitive Radio Oriented Wireless Networks (Crowncom16), May 2016, Grenoble, France. hal-01302652

HAL Id: hal-01302652

https://hal.inria.fr/hal-01302652

Submitted on 14 Apr 2016

HAL is a multi-disciplinary open access archive for the deposit and dissemination of scientific research documents, whether they are published or not. The documents may come from teaching and research institutions in France or abroad, or from public or private research centers.
L'archive ouverte pluridisciplinaire HAL, est destinée au dépôt et à la diffusion de documents scientifiques de niveau recherche, publiés ou non, émanant des établissements d'enseignement et de recherche français ou étrangers, des laboratoires publics ou privés. 


\title{
On the FPGA-based implementation of a flexible waveform from a high-level description: Application to LTE FFT case study
}

\author{
Mai-Thanh Tran, Matthieu Gautier, and Emmanuel Casseau \\ University of Rennes 1, IRISA, France, \\ \{mai-thanh.tran, matthieu.gautier, emmanuel.casseau\}@irisa.fr
}

\begin{abstract}
The Field Programmable Gate Array (FPGA) technology is expected to play a key role in the development of Software Defined Radio (SDR) platforms. To this aim, leveraging the nascent High-Level Synthesis (HLS) tools, a design flow from high-level specifications to Register-Transfer Level (RTL) description can be thought to generate processing blocks that can be reconfigured at run-time. Based on such a flow, this paper describes the architectural exploration of a Fast Fourier Transform (FFT) for Long Term Evolution (LTE) standard. Synthesis results show the tradeoff between reconfiguration time and area that can be achieved with such an approach.
\end{abstract}

Key words: High-Level Synthesis, Software Defined Radio, FPGA, LTE, Hardware implementation, Design flow.

\section{Introduction}

Advanced wireless communication standards are designed with various requirements in terms of data transmission rate, spectral efficiency and multiple channel bandwidths. To fulfil these requirements, many configurations of the waveform (PHY layer) features are allowed such as the number of antennas, the coding rate, the modulation scheme or the number of subcarriers in the case of Orthogonal Frequency Division Multiplexing (OFDM) modulation. In such a context, new needs of PHY layer implementation appear while the hardware implementation has to change from one configuration to one another in a short time, refereed to as run-time flexibility in this document.

An emergent technology that answers these new needs is Software Defined Radio (SDR) that allows both flexibility and fast prototyping capabilities from a high-level description [13]. However, when implementing the processing on Digital Signal Processors (DSP), SDR suffers from important power consumption and limited performance as compared to dedicated hardware fabrics. FPGA-based SDR is an old paradigm [8] offering a good tradeoff between reconfiguration capability and processing power. Fast prototyping capability of an FPGA-based SDR is achieved by leveraging High-Level Synthesis (HLS) principles and tools to generate Register-Transfer Level (RTL) descriptions from high-level specifications [18]. However, the issue of run-time flexibility is still opened. This 
paper discusses the FPGA-based implementation of a run-time hardware reconfiguration of a flexible waveform from its high-level description. The proposed methodology mainly aims at analyzing the performance of using a multi-mode processing block with control signals or Dynamic Partial Reconfiguration (DPR) to provide flexibility.

In this paper, the example of LTE standard is addressed. Among many configurations, this standard specifies that the computation of an OFDM symbol can be performed over several numbers of subcarriers among $\{128,256,512,1024$, $1536,2048\}$ and one symbol has a duration of $66.67 \mu \mathrm{s}$ [22]. Because Fast Fourier Transforms (FFTs) are used to compute the OFDM symbols, this paper discusses the FPGA implementation of a flexible FFT function that can operate with the different configurations of the LTE standard.

The main contributions of this paper are:

- To implement run-time reconfiguration from a high-level description of a processing block,

- To propose a flexible FFT implementation that covers the LTE configuration modes,

- To perform Design Space Exploration (DSE) of the proposed implementations using HLS capabilities.

The paper is organized as follows. A discussion over related works is given in Section 2. The flow to design a reconfigurable component from its high-level description is introduced in Section 3. Section 4 discusses the implementation of the flexible FFT for LTE purpose. RTL synthesis results and DPR performance for different reconfigurations are given in Section 5. Finally, conclusions and perspectives are drawn in Section 6.

\section{Related works}

Several proposals attempted to meet the flexibility requirements of an SDR by using software-based approaches. Indeed, software gives an abstraction level that enables more control over the hardware-based approaches. Two complementary approaches have been proposed namely, the SDR-specific languages to design the waveform [21][16][1] and the SDR middleware to provide the building environment [12][10]. They both take advantage of the abstraction level given by the software to achieve both compile-time and run-time flexibility.

Our proposal aims at keeping a high specification level while addressing FPGA platforms. To this end, HLS turns out to be a good candidate to achieve such a high abstraction level. The recent development of $H L S$ tools allows the consideration of components described in $\mathrm{C} / \mathrm{C}++$ languages. It raises the abstraction level compared to hardware languages like $V H D L$ and Verilog dedicated to RTL-based architectures. HLS fast prototyping capability enables the compile-time flexibility of an FPGA-based SDR [17]. 
There are two kinds of works that address the run-time flexibility of a FPGAbased SDR. The firsts propose to design multi-mode RTL components with control signals to switch between the different modes [5]. The others are based on DPR. Run-time DPR, refereed to as Hardware reconfiguration in the following, is the ability to reconfigure part of the FPGA (e.g. a functionality at the hardware level) while the rest of the FPGA continues to work. It is a research topic since the 90s [15] and it is now commonly used in FPGAs, since Xilinx and Altera provide such circuits [4][2]. The main advantages of the Hardware reconfiguration are to add hardware flexibility and to reuse hardware area, allowing power consumption and production cost reductions.

Based on the proposed methodology, a flexible FFT is proposed in this paper for LTE specifications. Hundreds of architectures for 128- to 2048-point FFT has been proposed by varying the degree of parallelism and the radix factorization [19][6]. These implementations are optimized in terms of speed, memory used and hardware logic requirements. A reconfigurable FFT for which algorithmic modifications allow the reuse of the resources while switching form one FFT to one another can be found in [22]. While all of these components are described at the RTL level so require very good skills in hardware design, our approach aims at providing a FFT design methodology from a high-level description to jointly achieve fast DSE and run-time reconfiguration.

\section{SDreconf: Design Flow for Software Defined Reconfiguration}

As mentioned in Section 2, there are different ways to achieve a flexible processing block while implementing it onto a FPGA. The first one is to design a multi-mode processing block and the second one is to use DPR (Hardware reconfiguration). In our approach, a multi-mode processing block can be described using dedicated algorithmic modifications of the processing block (Algorithmic reconfiguration) or with an automatic generation using a HLS encapsulation (Software reconfiguration). The goal of our design flow is to choose or combine these reconfigurations while describing the processing block at a high-level of description. This work is based on one commercially available HLS tool: VivadoHLS from Xilinx. It produces a RTL description of an application from its C-like specification. This section details the ways towards the generation of a flexible block.

Software reconfiguration: This reconfiguration uses HLS encapsulation to generate a Multi_MODE_BLOCK. The method uses the different modes of a BLOCK and generates a MULTI_MODE_BLOCK with a control input to switch between the modes. Algorithm 1 describes this encapsulation in the case of two modes BLOCK_A and BLOCK_B.

The advantages of this method are its simplicity, the rapid prototyping capability provided by HLS and the short reconfiguration time (one clock cycle). However, 


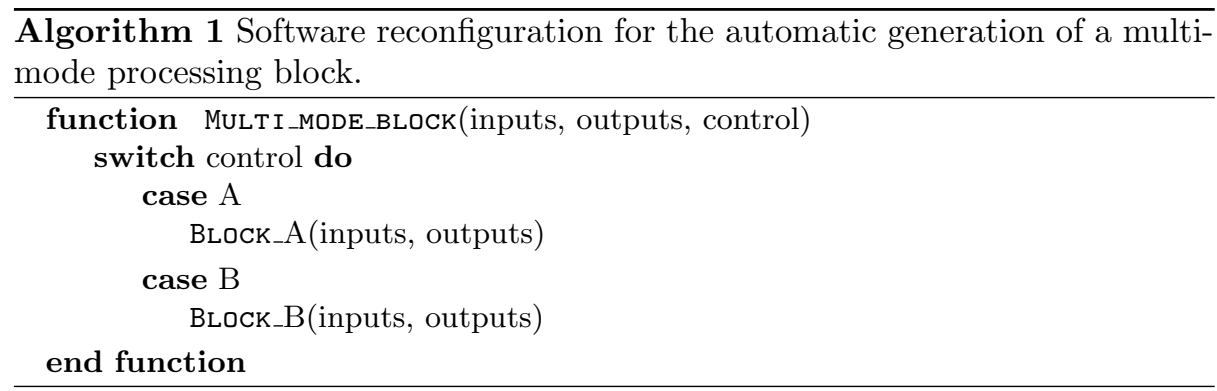

the resources can be important in that case. Actually the HLS tool does not share the resources efficiently although the modes are timewize mutually exclusive.

Hardware reconfiguration: In dynamic partial reconfiguration, the FPGA is divided into several regions being static (the areas that are not modified) or reconfigurable (the reconfigurable partitions). Each mode has its own partial bitstream. The partial bitstreams are stored in a memory and a software processor controls which partial bitstream is loaded into the reconfigurable partition at a particular time. The reconfigurable partition size must cover the area of the largest mode. Figure 1 shows the example of the Hardware reconfiguration of two modes BLOCK_A and BLOCK_B. The two modes are processed separately using first HLS and then RTL synthesis to generate two partial bitstreams. Part of this flow can be automated, at least to have an estimation of the performance. The main advantage is that the modes share the same area. The drawback is the reconfiguration time that depends on the size of the partial bitstream.

Algorithmic reconfiguration: For this kind of reconfiguration, the designer has to hand-code a dedicated processing block being intrinsically flexible. Signals are used to control the modes. Algorithmic optimizations should be done so that the HLS tool can share the resources between the modes.

Fig. 2 shows the design tradeoff between the resources and the reconfiguration time when the three kinds of reconfiguration are considered. Algorithmic reconfiguration is used to decrease the resources compared to Software reconfiguration and to decrease the reconfiguration time compared to Hardware reconfiguration. It provides the best performance in term of resources/reconfiguration time tradeoff. However, depending on the processing blocks, time to code the algorithmic reconfiguration can be important compared to Software reconfiguration.

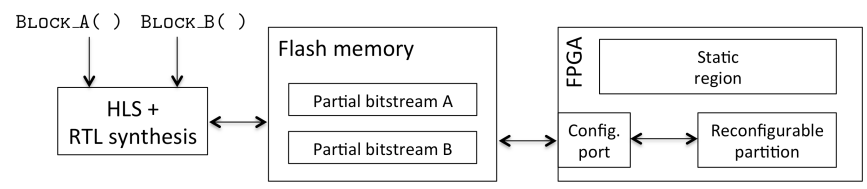

Fig. 1. Design approach based on hardware reconfiguration. 


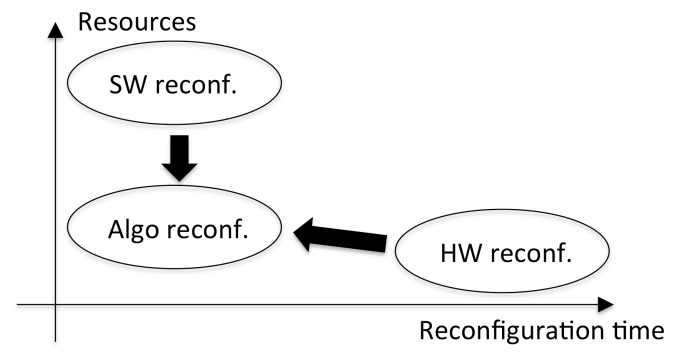

Fig. 2. Tradeoff between resources and reconfiguration time for the different reconfigurations.

Based on these three kinds of reconfiguration, the design flow used in this work is shown in Fig. 3. The different modes of a processing block can be provided by hand-coding or using a $H L S$ tool to generate different versions of a processing block by modifying synthesis constraints like throughput, latency, data size, etc. Such tools make it easy to explore a set of solutions via DSE [14][20][9] considering a given architecture.

In Fig. 3, Performance constraints are user-defined constraints such as resources/area, reconfiguration time, throughput or latency. The Performance analysis compares the performance of the three paths to the user-defined constraints. The basic idea is to first analyze the performance of the Software reconfiguration and Hardware reconfiguration paths and to use the Algorithmic reconfiguration if the Performance constraints are not met.

We have experimented this design flow with the rapid prototyping of a flexible FFT. An architecture exploration was performed allowing the comparison of the three kinds of reconfiguration.

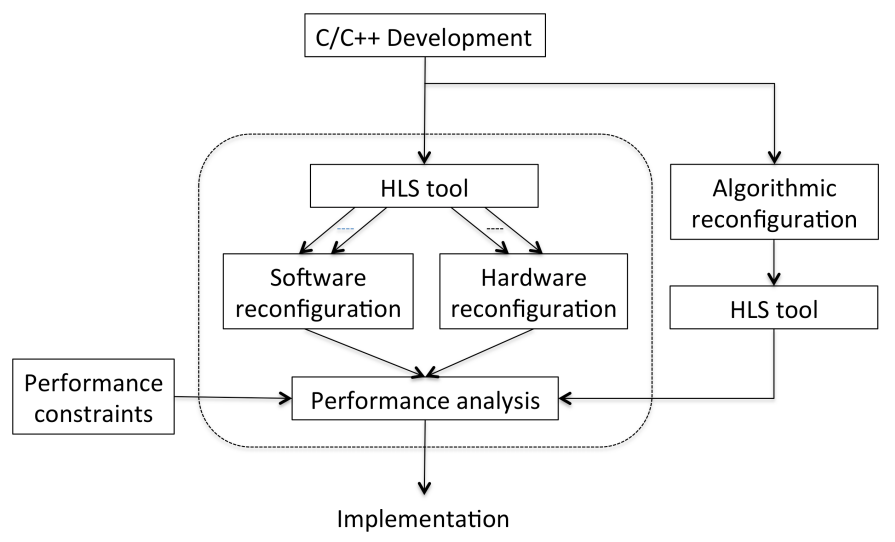

Fig. 3. Design flow for Software Defined Reconfiguration. 


\section{Building a flexible FFT}

In this section, a flexible FFT is designed using the proposed approach. Addressing LTE standard, the resulting FFT component should have six modes corresponding to these FFT sizes: $\{128,256,512,1024,1536,2048\}$. To introduce the different kinds of reconfiguration, preliminary results are first given in Sections 4.1 and 4.2 with the design of a flexible FFT with two sizes: $\{128,2048\}$. This design is based on two hand-coded FFT functions using the radix-2 Decimation-In-Time (DIT) algorithm which is the simplest and most common form of the Cooley-Tukey algorithm [7].

Experimental tools and setup: Vivado HLS 2013.3 is used for the high-level synthesis. DPR is setup with PlanAhead 14.6. The xc6vlx240tff1156 FPGA is targeted from Virtex 6 family as ML605 evaluation board will be used for future demonstration. Syntheses are based on a $100 \mathrm{MHz}$ clock frequency.

\subsection{Software reconfiguration}

First, two functions for 128- and 2048-point FFTs have been hand-coded and synthesized separately (BLOCK_FFT128( ) and BLOCK_FFT2048( ) respectively). Then the FFT with 2 modes (128/2048) has been designed using Software reconfiguration: using Algorithm 1, a function TwO_MODE_BLOCK ( ) is generated from BLOCK_FFT128( ) and BLOCK_FFT2048( ).

Table 1 shows the synthesis results and the latency (in number of clock cycles) of the three processing blocks. The resources are given with logical components such as the number of BRAM, DSP slices, LUT and FF.

As expected, BLOCK_FFT2048( ) requires more resources than BLock_FFT128( ): the number of BRAM to store cos and sin coefficients and input data is twofold and the number of DSP slices is 4 times more.

Except for the number of BRAM, Table 1 shows that the resources used by Two_Mode_BLock ( ) are (a little bit less than) the sum of the resources used by the two FFT blocks when synthesized separately. The HLS tool does not share the resources between the two functions although they are not executed at the same time. In this case, from the resources point of view, Software reconfiguration appears not to be an efficient solution to implement a flexible block. Latency is similar to mono-mode blocks.

\begin{tabular}{c||c|c|c} 
Processing block & BLOCK_FFT128( ) & BLOCK_FFT2048( ) & TWO_ModE_BLOCK( ) \\
\hline \hline BRAM & 6 & 12 & 12 \\
\hline DSP & 17 & 65 & 82 \\
\hline LUT & 1017 & 2522 & 3459 \\
\hline FF & 862 & 2443 & 3241 \\
\hline \hline Latency & 5362 & 72410 & $5491 / 72411$
\end{tabular}

Table 1. Performance of Software reconfiguration for a FFT with 2 modes (128/2048). 


\begin{tabular}{|c|c|c|c|c|c|}
\hline & \multicolumn{2}{|c|}{$\begin{array}{l}\text { Processing block: } \\
\text { Resources needed }\end{array}$} & \multicolumn{3}{|c|}{$\begin{array}{c}\text { Partition: } \\
\text { Resources used }\end{array}$} \\
\hline & FFT 128 & FFT 2048 & FFT 128 & FFT 2048 & FFT 128/2048 \\
\hline BRAM & 6 & 12 & 6 & 17 & 17 \\
\hline DSP & 17 & 65 & 24 & 68 & 68 \\
\hline LUT & 1017 & 2522 & 1440 & 4080 & 4080 \\
\hline $\mathrm{FF}$ & 862 & 2443 & 2880 & 8160 & 8160 \\
\hline Bitstream size & $\mathrm{n} / \mathrm{a}$ & $\mathrm{n} / \mathrm{a}$ & 138672 Bytes & 416016 Bytes & $2 \times 416016$ Bytes \\
\hline Reconf. time & $\mathrm{n} / \mathrm{a}$ & $\mathrm{n} / \mathrm{a}$ & $10.98 \mathrm{~ms}$ & $32.9 \mathrm{~ms}$ & $32.9 \mathrm{~ms}$ \\
\hline Latency & 5362 & 72410 & 5362 & 72410 & $5362 / 72410$ \\
\hline
\end{tabular}

Table 2. Performance for Hardware reconfiguration of a FFT with 2 modes (128/2048).

\subsection{Hardware reconfiguration}

The two functions BLock_FFT128( ) and BLock_FFT2048( ) are now used for Hardware reconfiguration. In Xilinx's FPGAs [4], the functions to be dynamically placed are mapped into an area called a reconfigurable partition. Generally speaking, a partition has a rectangle shape and uses resources according to this area even if they are not needed.

Two partitions have been first generated using PlanAhead tool: one for the FFT 128 only and one for the FFT 2048 only. Table 2 shows the resources used by these partitions. Bitstream sizes are also given. Because FFT 128's partition uses less logical components than FFT 2048's one, it is smaller than FFT 2048's one. The reconfiguration time depends on the bitstream size so the reconfiguration time for FFT 128 is smaller than FFT 2048's one. Reconfiguration time is computed from PRCC tool (Partial Reconfiguration Cost Calculator) from Technical University of Crete [3] assuming that the reconfiguration controller is an on-chip MicroBlaze processor ${ }^{1}$.

For comparison, Table 2 also shows the resources needed by FFT 128 and FFT 2048 when they are placed as static logic (i.e. not as a reconfigurable module, so resources needed are same as in Table 1 ).

To perform the DPR of the 2 functions, a third partition called FFT 128/2048 in Table 2 has been defined. In this case, the two functions are placed on the same partition, i.e. on the same area of the FPGA. For each type of logical component, the resources used by this partition are based on the more costly case. In our case, FFT 2048 partition always needs the largest number of resources whatever the kind of logical component. Thus the resulting FFT 128/2048 partition is based on the FFT 2048 partition.

Reconfigurable partition's latency is equal to the latency of the function when synthesized alone onto a static region. The hardware reconfiguration needs $32.9 \mathrm{~ms}$ to switch from one mode to the other one whereas only one clock cycle is required with Software reconfiguration. It means many OFDM symbols will be lost in practice when changing the mode with hardware reconfiguration.

\footnotetext{
${ }^{1}$ Higher throughput up to 400 MBytes/s may be reached using a dedicated controller
} so that reconfiguration time can be reduced. 


\subsection{Algorithmic reconfiguration}

The FFT for LTE standard should have six modes: $\{128,256,512,1024,1536,2048\}$. Based on the previous results, a Software reconfiguration will generate a huge component as the resources are not shared. Hardware reconfiguration makes resource sharing possible but may require a long reconfiguration time. In this section, we first present a power-of-two point FFT for algorithmic reconfiguration to share the resources between its different modes. Then, a FFT 1536 function is presented. Sharing the resources of these 2 functions is discussed.

Power-of-two point FFT for algorithmic reconfiguration: The power-of-two point FFT has 5 modes $\{128,256,512,1024,2048\}$. A dedicated control signal is used to decide the mode and the HLS tool deals with the FFT_size as a variable. Indeed, as presenting in Algorithm 2, FFT_size and FFT_stages are calculated based on the control signal value. When FFT_size and FFT_stages are determined, a standard three-loop structure for the FFT based on radix-2 is computed. The first loop determines the stage. The second loop chooses butterflies with the same twiddle factor at each stage. Last loop computes all the chosen butterflies.

This algorithmic reconfiguration generates a BLOCK_FFTPow2() function with only one main FFT core for the five different modes. With this function, the resources should be approximately the ones used by the largest FFT (i.e. 2048).

1536-point FFT: By applying the Cooley-Tukey algorithm [7] for a FFT size of 1536, the BLOCK_FFT1536( ) function can be generated using three BLOCK_FFT512( ) functions and one radix-3 function [11] as shown in Fig 4. First, the 1536 inputs of the FFT are split into three parts. Those parts are computed as three 512-point FFTs independently. Then, while the first part is kept as it is, the second and the third ones are multiplied by twiddles factors. Last, the radix-3 function is applied to compute the final results.

Resource sharing may theoretically be done between BLock_FFT512( ) functions of the 1536-point FFT and the power-of-two point FFT because they are

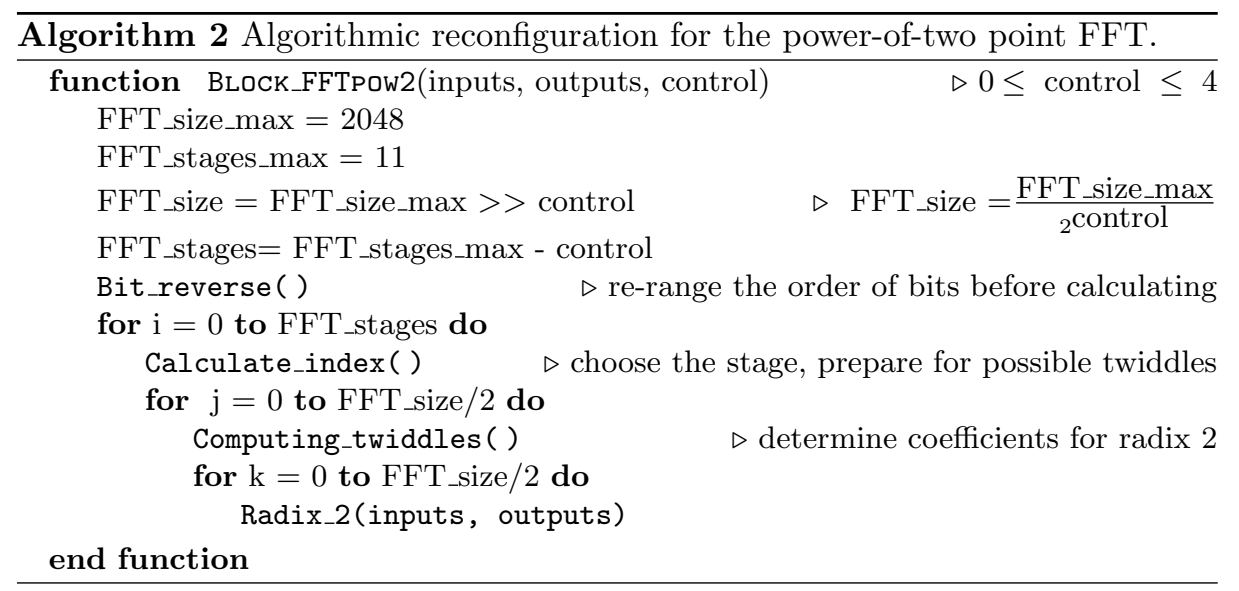




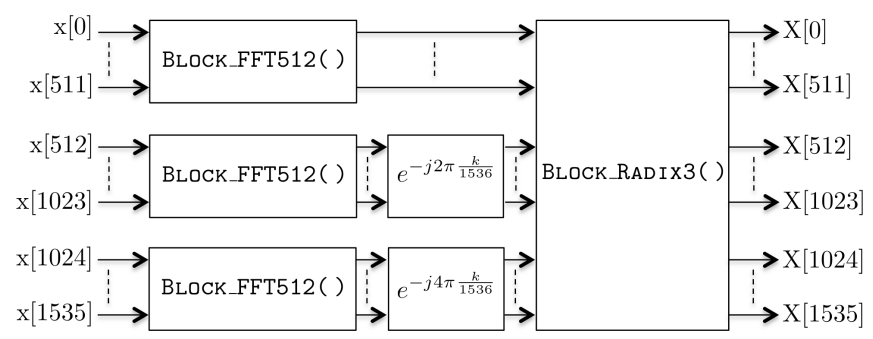

Fig. 4. Functional description of BLock_FFT1536( ) function.

both based on the radix-2 algorithm. On the contrary, the BLock_RADIX3( ) function is based on radix-3 thus it can not share resources.

\section{Performance results}

This section presents the architectural exploration of a flexible FFT for LTE standard. First, design space will be explored for the function BLock_FFTPOW2( ). Then, the combination between BLock_FFTpow2( ) and BLock_FFT1536( ) will be addressed using Software and Hardware reconfigurations.

\subsection{Design space exploration of the power-of-two point FFT}

In order to generate a flexible FFT that respects the design constraints (area, latency, throughput, ...), HLS allows the DSE of a processing block by using compilation directives. This part presents the DSE of the function BLock_FFTPOW2(). Several directives are made available on a typical HLS tool (e.g. memory mapping, pipeline, loop unrolling, inlining, ...). They make it possible to optimize the design for area or latency. In this study, because of the loop structures and the data dependencies of the FFT, loop unrolling is used. Loop unrolling reduces the total loop iterations by duplicating (with a factor $\mathrm{U}$ ) the loop body so that

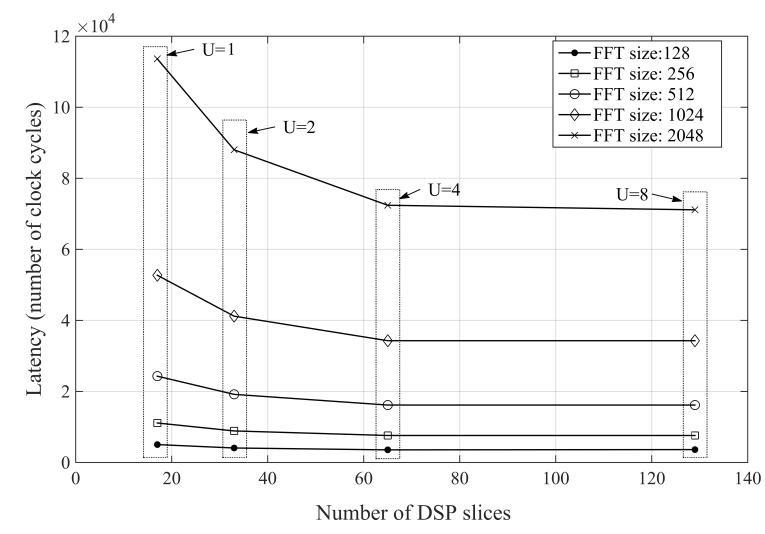

Fig. 5. DSE of BLOCK_FFTPOW2( ) function. 
we can tradeoff between area and latency.

Figure 5 shows the latency of BLock_FFTPOw2( ) processing block as a function of the number of DSP slices. In practice, four multi-mode components have been generated by varying the unroll factor U. Each component is characterized by its numbers of DSP slices. The number of DSP slices increases with the unroll factor while the latency decreases with the unroll factor. One's can see a floor effect appears in Figure 5. Due to the BRAM accesses (read and write data from/to memory) that reach their bounds for $\mathrm{U}=4$, increasing the number of DSP resources is not useful in practice.

The same behavior is also obtained for the number of LUT and FF. Thus $U=4$ seems to be a good tradeoff between number of resources and latency.

\subsection{Proposed flexible FFT implementations for LTE standard}

Performance of the multi-mode FFT with software reconfiguration: Software reconfiguration is applied first to design the FFT with 6 modes for LTE. Using Algorithm 1, a MuLti_Mode_BLOCK_LTE( ) function is generated from the two functions BLock_FFTpow2( ) and BLock_FFT1536( ). Table 3 shows the synthesis results and latency (in number of clock cycles) of the three processing blocks. $\mathrm{U}=4$ is used for BLOCK_FFTpow2( ). The resources used by Multi_Mode_Block_LTE( ) are almost the sum of the resources used by BLOCK_FFTPOW2( ) and BLock_FFT1536( ) when synthesized separately. As observed in Section 4.1, the HLS tool does not share the resources between the two functions even if they are not executed at the same time.

Performance of the multi-mode FFT with hardware reconfiguration: Hardware reconfiguration is now applied on the two functions BLock_FFTpow2( ) and BLOCK_FFT1536 ( ). Two partitions are first generated: one for the power-of-two point FFT only and one for the FFT 1536 only. Then, a partition is finally created for the DPR of the 2 FFTs.

Table 4 shows the synthesis results. The partition for FFT 1536 is smaller than the power-of-two point FFT's one. Actually, number of BRAMs is greater but function BLOCK_FFT1536( ) uses less DSP slices than BLock_FFTPow2() so that its area is smaller. Thus, when combining the 2 FFTs into one partition, the resulting partition is based on power-of-two point FFT's partition.

\begin{tabular}{c||c|c|c} 
Processing block & BLock_FFTPOW2 ( ) & BLOCK_FFT1536( ) & Mult I_ModE_BLOCK_LTE( ) \\
\hline \hline BRAM & 12 & 14 & 26 \\
\hline DSP & 65 & 40 & 103 \\
\hline LUT & 2553 & 3054 & 5256 \\
\hline FF & 2497 & 2010 & 4299 \\
\hline \hline Latency & cf. Fig.5-U=4 & 52198 & cf. Fig.5/52198
\end{tabular}

Table 3. Performance of a FFT for LTE standard with Software reconfiguration. 


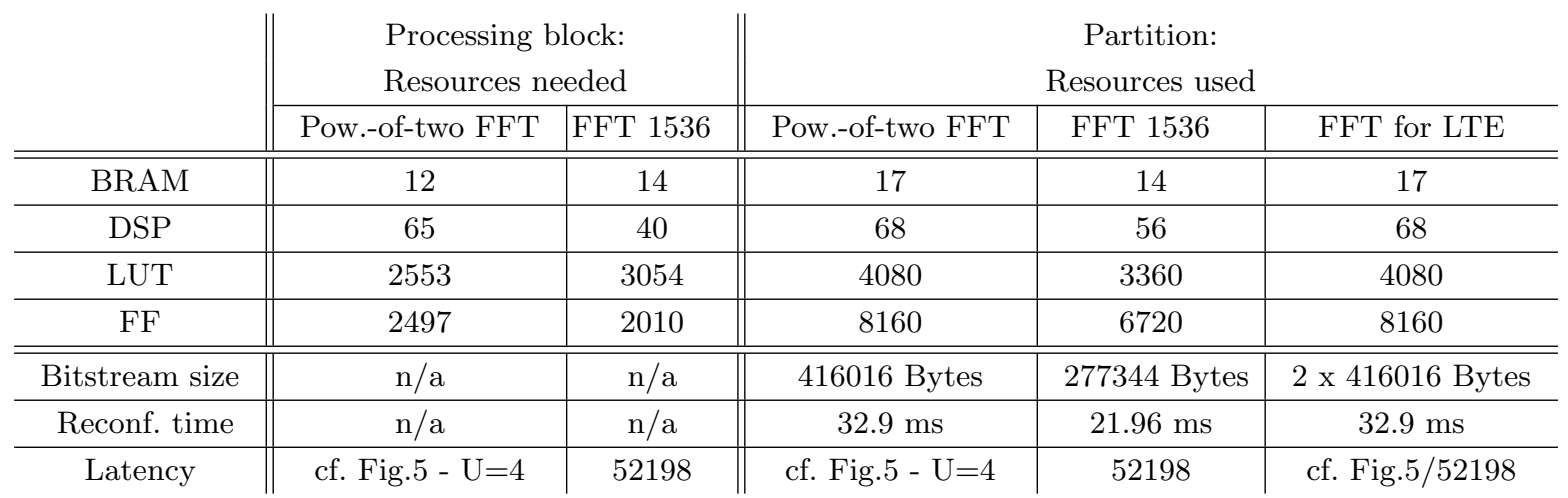

Table 4. Performance of a FFT for LTE standard with Hardware reconfiguration.

Compared with Software reconfiguration, the multi-mode FFT based on hardware reconfiguration uses less resources (BRAM and DSP are the more area costly logical components). When the FFT size has to be modified but is still a power of two, in both cases only one clock cycle is required to reconfigure. However, $32.9 \mathrm{~ms}$ are required to reconfigure when switching from a 1536-point FFT and a power-of-two point FFT (or vice versa) with hardware reconguration whereas only one clock cycle is required with Software reconfiguration.

\section{Conclusion}

This paper presents a methodology for the implementation of run-time reconfiguration in the context of FPGA-based SDR. The proposed design flow allows the exploration between dynamic partial reconfiguration and control signal based multi-mode design. This architectural tradeoff relies upon HLS and its associated design optimizations.

A flexible FFT for LTE standard is implemented as a case study. The proposed component combines both DPR (to deal with FFT size of 1536) and algorithmic reconfiguration (when FFT size is a power of two). Synthesis results show the tradeoff that could be achieved between the reconfiguration time and the FPGA resource utilization. Future work is to explore the implementation of other processing functions and the automation of the design flow.

\section{References}

1. GNU Radio: The free and open software radio ecosystem. www.gnuradio.org.

2. Increasing Design Functionality with Partial and Dynamic Reconfiguration in 28nm FPGAs, Altera white paper, WP-01137-1.0, www.altera.com.

3. Kyprianos Papadimitriou, Microprocessor and Hardware Laboratory, Technical University of Crete. Partial Reconfiguration Cost Calculator. URL: http://users. isc.tuc.gr/ kpapadimitriou/prcc.html.

4. Partial Reconfiguration User Guide, UG702 (v14.1), www.xilinx.com, 2012.

5. E. Casseau and B. Le Gal. Design of Multi-Mode Application-Specific Cores Based on High-Level Synthesis. Integration, the VLSI Journal, Elsevier, 45(1):9-21, 2012. 
6. Jienan Chen, Jianhao Hu, Shuyang Lee, and G.E. Sobelman. Hardware Efficient Mixed Radix-25/16/9 FFT for LTE Systems. Very Large Scale Integration (VLSI) Systems, IEEE Transactions on, 23(2):221-229, February 2015.

7. James W. Cooley and John W. Tukey. Mathematics of Computation, chapter An algorithm for the machine calculation of complex Fourier series, pages 297-301. American Mathematical Society, 1965.

8. M. Cummings and S. Haruyama. FPGA in the Software Radio. IEEE Communications Magazine, 37(2):108-112, February 1999.

9. M. Gautier, G.S. Ouedraogo, and O. Sentieys. Design Space Exploration in an FPGA-Based Software Defined Radio. In Euromicro Conference on Digital System Design (DSD), pages 22-27, Verona, Italy, August 2014.

10. Antoni Gelonch, Xavier Revs, Vuk Marojevik, and Ramon Ferrús. P-HAL: a Middleware for SDR applications. SDR Forum Technical Conference, November 2005.

11. Freescale Semiconductor Incorporated. Software Optimization of DFTs and IDFTs Using the StarCore SC3850 DSP Core. Application Note AN3980, 2009.

12. G. Jianxin, Y. Xiaohui, G. Jun, and L. Quan. The Software Communication Architecture: Evolutions and Trends. IEEE Conference on Computational Intelligence and Industrial Applications (PACIIA), November 2009.

13. J. F. Jondral. Software-defined radio: Basics and evolution to cognitive radio. EURASIP Journal on Wireless Communications and Networking, 3, 2005.

14. Y. Le Moullec, J.-P. Diguet, N. Ben Amor, T. Gourdeaux, and J.-L. Philippe. Algorithmic-level specification and characterization of embedded multimedia applications with design trotter. Journal of VLSI signal processing systems for signal, image and video technology, 42(2):185-208, 2006.

15. E. Lemoine and D. Merceron. Run time reconfiguration of FPGA for scanning genomic databases. In IEEE Symposium on FPGAs for Custom Computing Machines, pages 90-98, April 1995.

16. Y. Lin, R. Mullenix, M. Woh, S. Mahlke, T. Mudge, A. Reid, and K. Flautner. SPEX: A Programming Language for Software Defined Radio. In Software Defined Radio Technical Conference and Product Exposition (SDR-Forum), November 2006 .

17. G.-S. Ouedraogo, M. Gautier, and O. Sentieys. A frame-based DomainSpecific Language for rapid prototyping of FPGA-based Software-Defined Radios. EURASIP Journal on Advances in Signal Processing, 2014(1):164, November 2014.

18. G. S. Ouedraogo, M. Gautier, and O. Sentieys. Frame-based Modeling for Automatic Synthesis of FPGA-Software Defined Radio. IEEE International Conference on Cognitive Radio Oriented Wireless Networks and Communications (CROWNCOM), June 2014.

19. T. Pitkanen and J. Takala. Low-power application-specific processor for FFT computations. In IEEE International Conference on Acoustics, Speech and Signal Processing (ICASSP), pages 593-596, April 2009.

20. B. So, M. W. Hall, and P. C. Diniz. A compiler approach to fast hardware design space exploration in FPGA-based systems. In Proceedings of the ACM SIGPLAN Conference on Programming language design and implementation (PLDI), pages 165-176, New York, USA, 2002.

21. E. D. Willink. The Waveform Description Language: Moving from Implementation to Specification. IEEE Military Communications Conference (MILCOM), 2001.

22. Chia-Hsiang Yang, Tsung-Han Yu, and D. Markovic. Power and Area Minimization of Reconfigurable FFT Processors: A 3GPP-LTE Example. Solid-State Circuits, IEEE Journal of, 47(3):757-768, March 2012. 